\title{
Desempenho de vacas de corte Purunã submetidas a diferentes manejos de amamentação
}

\author{
Ivan César Furmann Moura(1), Fernando Kuss(2), José Luis Moletta(3), Luis Fernando Glazenapp de Menezes(2), \\ Douglas Sampaio Henrique( ${ }^{(2)}$, Leandro Cavalcante Lipinski ${ }^{(1)}$ e Adriana de Souza Martins ${ }^{(1)}$
}

\begin{abstract}
(1)Universidade Estadual de Ponta Grossa, Avenida General Carlos Cavalcanti, no 4.748, Uvaranas, CEP 84030-900 Ponta Grossa, PR, Brasil. E-mail: ivancesar.moura@bol.com.br, leandrolipinski@yahoo.com.br, dri261@yahoo.com.br (2)Universidade Tecnológica Federal do Paraná, Campus Dois Vizinhos, Estrada para Boa Esperança, Km 04, CEP 85660-000 Dois Vizinhos, PR, Brasil. E-mail: fernandokuss@utfpr.edu.br, luismenezes@utfpr.edu.br, douglas@utfpr.edu.br ${ }^{(3)}$ Instituto Agronômico do Paraná, Avenida Euzébio de Queiróz, s/noo, Uvaranas, Caixa Postal 129, CEP 84001-970 Ponta Grossa, PR, Brasil. E-mail: moletta@iapar.br
\end{abstract}

Resumo - O objetivo deste trabalho foi avaliar o desempenho ponderal e reprodutivo de vacas de corte submetidas a diferentes manejos de amamentação. Durante a estação de monta, de 85 dias, foram avaliadas 161 vacas de corte da raça Purunã, de acordo com os seguintes manejos de amamentação: desmame precoce, vacas separadas dos seus bezerros aos 75 dias pós-parto; amamentação controlada, vacas separadas de seus bezerros aos 75 dias de idade, mas colocadas para amamentar uma vez ao dia durante a estação de monta; e desmame convencional, vacas mantidas com seus bezerros ao pé até o final da estação de monta, aos 160 dias de idade dos bezerros, em média. As taxas de prenhez não foram significativamente afetadas pelos manejos de amamentação, tendo sido de $97 \%$ no desmame precoce, de $96 \%$ na amamentação controlada e de $90 \%$ no desmame convencional. No entanto, o desmame precoce resultou em menor eficiência reprodutiva $(28,26 \mathrm{~kg})$, quando comparado à amamentação controlada $(35,09 \mathrm{~kg})$ e ao desmame convencional $(35,34 \mathrm{~kg})$. Vacas de corte mantidas em boas condições corporais ao parto e ao início da estação de monta apresentam alta taxa de fertilidade, independentemente do manejo de amamentação dos bezerros.

Termos para indexação: amamentação controlada, anestro pós-parto, desmame precoce, eficiência reprodutiva, intervalo entre partos, taxa de fertilidade.

\section{Performance of Purunã beef cows subjected to different nursing managements}

Abstract - The objective of this work was to evaluate the weight and reproductive performance of beef cows subjected to different nursing managements. During the breeding season of 85 days, 161 beef cows of the Purunã breed were evaluated, according to the following feeding managements: early weaning, cows separated from their calves at 75 days postpartum; controlled nursing, cows separated from their calves at 75 days of age, but taken to nurse once a day during the breeding season; and conventional weaning, cows kept with their calves until the end of the breeding season, at 160 days of age of calves, in average. Fertility rates were not significantly affected by weaning managements, being $97 \%$ for early weaning, $96 \%$ for controlled nursing, and $90 \%$ for conventional weaning. However, early weaning resulted in lower reproductive efficiency $(28.26 \mathrm{~kg})$, when compared to controlled nursing $(35.09 \mathrm{~kg})$ and conventional weaning $(35.34 \mathrm{~kg})$. Beef cows maintained in good body conditions at birth and at the beginning of the breeding season have a high fertility rate, regardless of calf-feeding management.

Index terms: controlled nursing, post-partum anestrus, early weaning, reproductive efficiency, calving interval, fertility rate.

\section{Introdução}

Para alcançar a máxima eficiência produtiva em vacas de corte, deve-se manter o intervalo entre partos próximo de um ano (Borges \& Gregory, 2003). Para isso, o intervalo parto-concepção não deve ser superior a 85 dias, considerando-se um período de gestação de aproximadamente 280 dias.
Conforme Godoy et al. (2004), a duração do anestro pós-parto é afetada por diversos fatores, entre os quais destacam-se o manejo nutricional e a amamentação, além de outros como estação do ano, raça, idade e presença do touro. Quando não há evidências de deficiência nutricional nas vacas, o anestro pós-parto está fortemente ligado à frequência, à intensidade e à duração da amamentação (Grecellé et al., 2006). 
A partir desse conceito, foram desenvolvidas técnicas para melhorar o desempenho reprodutivo de vacas no puerpério, como o desmame precoce, a amamentação controlada, o desmame interrompido e a suplementação de bezerros. Em vacas cujos bezerros foram desmamados aos 76 dias de idade, Vaz et al. (2010a) verificaram incremento de 30 pontos percentuais na taxa de prenhez (de 55,5 para $86,34 \%$ ), em comparação às que tiveram seus bezerros desmamados aos 148 dias de idade.

Pencai et al. (2011) avaliaram a amamentação controlada, em que os bezerros somente têm acesso a suas mães durante $30 \mathrm{~min}$ por dia, durante a estação de monta. Os autores observaram efeito significativo da amamentação controlada sobre a taxa de prenhez, que aumentou de 54,5 para 87,3\%, quando comparada à do manejo de amamentação convencional (vacas com bezerros permanentemente ao pé das mães, até aos 210 dias).

Qualquer mudança tecnológica na pecuária de corte para cria deve ser feita com o objetivo de aumentar a taxa de natalidade (Menegaz et al., 2008), e os manejos de amamentação podem ter impacto significativo sobre as taxas reprodutivas.

O objetivo deste trabalho foi avaliar o desempenho ponderal e reprodutivo de vacas de corte submetidas a diferentes manejos de amamentação, durante a estação de monta de 85 dias.

\section{Material e Métodos}

O experimento foi realizado na Estação Experimental da Fazenda Modelo, do Instituto Agronômico do Paraná (Iapar), localizada no Município de Ponta Grossa, PR $\left(25^{\circ} 6^{\prime} \mathrm{S}, 50^{\circ} 9^{\prime} \mathrm{W}\right.$, a $783 \mathrm{~m}$ de altitude). Conforme a classificação de Köppen, o clima da região é do tipo $\mathrm{Cfb}$, temperado sem estação seca, com temperatura média anual de $17,6^{\circ} \mathrm{C}$, média das máximas de $24,3^{\circ} \mathrm{C}$ e média das mínimas de $8,5^{\circ} \mathrm{C}$. A precipitação média anual é de $1.400 \mathrm{~mm}$, com chuvas mais frequentes no período de primavera/verão e mais escassas no outono. O solo é classificado como Latossolo Vermelho-Escuro, de textura média.

O trabalho foi desenvolvido entre junho de 2011 e fevereiro de 2012, período que englobou a estação de nascimentos (junho, julho e agosto), a estação de monta (setembro, outubro e novembro) e o diagnóstico de gestação. Foram utilizadas 161 vacas e seus respectivos bezerros, oriundos da raça Purunã (40\% Charolês, 25\% Aberdden Angus, 25\% Caracu e 10\% Nelore). Avaliaram-se três categorias de vacas: jovens (primíparas), com idade de até 43 meses; adultas (duas a cinco crias), com idade de 44-90 meses; e velhas (acima de seis crias), com idade superior a 90 meses.

As vacas foram submetidas aos seguintes manejos de amamentação: desmame precoce, vacas separadas dos seus bezerros aos 75 dias pós-parto; amamentação controlada, vacas separadas dos bezerros a partir dos 75 dias de idade, mas colocadas para amamentar uma vez ao dia, durante o período de estação de reprodução (85 dias); desmame convencional, vacas mantidas com seus bezerros ao pé, os quais eram amamentados até o fim da estação de monta e desmamados com 160 dias de idade.

A distribuição dos animais em seus respectivos tratamentos foi realizada em duas etapas, para possibilitar o manejo de desmame precoce e a amamentação controlada em bezerros com idade média de 75 dias. A primeira etapa foi realizada no início da estação de monta ( 1 de setembro) com as vacas paridas durante junho. A segunda etapa se deu no início do segundo mês de estação de monta (1 de outubro), com as vacas paridas no segundo mês da estação de nascimento (julho). As vacas que pariram no último mês da estação de parição (agosto) não foram avaliadas, por apresentar número pequeno e desproporcional de animais, para divisão entre períodos.

Durante o período entre nascimento e início da estação de monta, que antecedeu à divisão dos tratamentos, as vacas ocuparam um único lote, de pastagem composta por aveia-branca (Avena sativa L.) e triticale (X Triticosecale Wittmack). Após a separação dos lotes em seus respectivos tratamentos, as vacas e os bezerros submetidos ao tratamento com amamentação controlada foram retirados do lote do rebanho geral e mantidos em piquete exclusivo. Os bezerros foram separados em instalações apropriadas, para impedir que alcançassem suas mães. As vacas do desmame precoce permaneceram na mesma área das vacas do desmame convencional, uma vez que ambos os grupos não necessitavam de manejos intensivos como as vacas do tratamento amamentação controlada.

As áreas de pastagem da fazenda encontravam-se em sistema de integração lavoura-pecuária. No início do plantio da lavoura de primavera/verão, na segunda quinzena de outubro, as vacas foram remanejadas 
para área composta de pastagem natural e Hemarthria florida, tendo sido mantidas nesses campos sob pastejo contínuo com taxa de lotação fixa, até o final do período experimental. Nessas áreas, as vacas de todos os grupos tinham acesso à água natural e a cochos de sal mineralizado à vontade.

A partir do início da estação de monta, todas as vacas foram submetidas a pesagens periódicas e à avaliação do escore de condição corporal (ECC), segundo a metodologia de Lowman et al. (1973), em que: 1, estado muito magro; 2, magro; 3 , médio; 4, gordo; e 5, muito gordo. Essas avaliações foram realizadas aproximadamente a cada 30 dias, e a pontuação de escore foi realizada sempre pelo mesmo avaliador, para manter a mesma calibração visual entre uma avaliação e outra.

As estimativas da disponibilidade de forragem (Tabela 1) foram realizadas a cada 30 dias, pelo método comparativo (Haydock \& Shaw, 1975). Foram realizados cinco cortes de $0,5 \mathrm{~m}^{2}$ rentes ao solo, que receberam pontuação de 1 (menor disponibilidade forrageira) a 5 (máxima disponibilidade forrageira). Foram realizadas 30 avaliações visuais aleatórias que receberam pontuação de 1 a 5 , de acordo com os cortes previamente feitos nos piquetes. As amostras foram acondicionadas em sacos de papel identificados conforme a área e a data de coleta, pesadas para obter a massa de matéria verde, mantidas em estufa de ventilação forçada de ar a $60^{\circ} \mathrm{C}$ por 72 horas, e novamente pesadas para determinação da massa de

Tabela 1. Disponibilidade e qualidade da forragem utilizada na manutenção de vacas de corte Purunã, submetidas aos diferentes manejos de amamentação.

\begin{tabular}{|c|c|c|c|}
\hline \multirow[t]{2}{*}{ Características avaliadas $^{(1)}$} & \multicolumn{3}{|c|}{ Épocas de avaliação ${ }^{(2)}$} \\
\hline & IPR & MPR & FPR \\
\hline Disponibilidade de forragem ( $\mathrm{kg} \mathrm{ha}^{-1}$ de MS) & 1.650 & 1.300 & 575 \\
\hline Pressão de pastejo (kg de $\mathrm{PV} \mathrm{kg}^{-1}$ de MS) & 0,55 & 0,44 & 1,14 \\
\hline Taxa de lotação (UA ha-1) & 1,56 & 1,35 & 1,33 \\
\hline MS da forragem (\%) & 25,88 & 47,28 & 30,00 \\
\hline Proteína bruta (\% de MS) & 6,58 & 7,38 & 6,52 \\
\hline FDN (\% de MS $)$ & 64,98 & 64,52 & 69,50 \\
\hline FDA (\% de MS) & 39,95 & 45,44 & 40,24 \\
\hline Extrato etéreo (\% de MS) & 3,26 & 2,70 & 1,79 \\
\hline Cinzas totais $(\%$ de MS $)$ & 6,64 & 6,36 & 7,37 \\
\hline NDT $(\%$ de MS $)$ & 60,54 & 58,87 & 58,40 \\
\hline
\end{tabular}

${ }^{(1)} \mathrm{MS}$, matéria seca; PV, peso vivo; FDN e FDA, fibra em detergente neutro e ácido, respectivamente; NDT, nutrientes digestíveis totais. ${ }^{(2)}$ IPR, início do período reprodutivo; MPR, meio do período reprodutivo; FPR, final do período reprodutivo. matéria parcialmente seca. As amostras foram moídas em peneira de $1 \mathrm{~mm}$, armazenadas em sacos de plástico apropriados, identificadas e enviadas ao Laboratório de Bromatologia da Universidade Tecnológica Federal do Paraná, Campus Dois Vizinhos, para realização das análises laboratoriais.

Foram determinados os dados requeridos para uma descrição precisa do conteúdo de energia da forragem, segundo o método proposto pelo National Research Council (2001). A partir dos resultados das análises bromatológicas da forragem, o conteúdo de nutrientes digestíveis totais (NDT) foi estimado por meio da equação proposta originalmente por Weiss et al. (1992): $\mathrm{NDT}=\mathrm{PBD}+2,25 \times$ AGD + FDNcpD + CNFD, em que: PBD, AGD, FDNcpD e CNFD são, respectivamente, proteína bruta digestível, ácido graxo digestível, fibra em detergente neutro (isenta de cinzas e proteína) digestível e carboidratos não fibrosos digestíveis.

Os teores de digestibilidade de cada nutriente utilizado para a realização da equação foram determinados conforme Weiss et al. (1992) e National Research Council (2001): $\mathrm{PBD}=\mathrm{PB} \times \exp [-1,2 \times(\mathrm{PIDA} / \mathrm{PB})]$; $\mathrm{CNFD}=0,98(100-[(\mathrm{FDN}-\mathrm{PIDN})+\mathrm{PB}+\mathrm{EE}+\mathrm{MM}]) \times \mathrm{FAP}$, em que, FAP é o fator de ajuste para processamento físico, equivalente a 1 , de acordo com o National Research Council (2001); AGD = $(\mathrm{EE}-1) \times 100$; FDNcpD $=0,75($ FDNcp - LIG $) \times\left[1-(\mathrm{LIG} / \mathrm{FDNp})^{0,667}\right]$.

$\mathrm{O}$ sistema de acasalamento utilizado durante $\mathrm{o}$ período reprodutivo foi o de monta natural, com uso de touros Purunã, na relação de 1:30 (um touro para trinta vacas). O diagnóstico de gestação das vacas foi realizado 60 dias após o encerramento da estação de monta (final de janeiro), por meio de ultrassonografia transretal, com uso de aparelho com câmera, modelo Aloka Echo SSD-500 (Hitachi Aloka, Tóquio, Japão). Além do diagnóstico de gestação, foi estimada a idade do feto, com intervalo aproximado de 23 dias. No dia da realização do diagnóstico, havia fetos com idade igual a $60-83,84-106,107-129$ e 130-152 dias. Com isso, foi possível estimar o período de concepção das vacas, isto é, o intervalo parto-concepção, já que cada intervalo de idade do feto (dias) seria o período de concepção da vaca: de 0 a 23, 24 a 46, 47 a 69 e 70 a 92 dias a partir do início da estação de monta.

Os bezerros foram pesados a cada 30 dias durante a estação de monta, tendo-se utilizado o peso de 
desmame aos 160 dias de idade. Para a determinação das características de índice de produção de bezerro e eficiência produtiva das vacas, seguiu-se o modelo utilizado por Vaz et al. (2010b).

Utilizou-se o delineamento experimental inteiramente casualizado. As análises foram realizadas com auxílio do procedimento GLM, e os dados foram analisados pelo programa SAS, versão 8.1 (SAS Institute, Cary, NC, EUA). O desempenho das vacas foi avaliado pelo teste de Tukey, a 5\% de probabilidade. A taxa de prenhez foi analisada pelo teste do qui-quadrado, com uso do procedimento GLM (SAS Institute, Cary, NC, EUA), segundo o modelo: $\mathrm{Y}_{\mathrm{ijk} l}=\mu+\mathrm{T}_{\mathrm{i}}+\mathrm{P}_{\mathrm{j}}+\mathrm{C}_{\mathrm{k}}+\mathrm{S}_{1}+\left(\mathrm{T}^{*} \mathrm{C}\right)_{\mathrm{ik}}+\varepsilon_{\mathrm{ijk} \mathrm{l}}$, em que $\mathrm{Y}_{\mathrm{ijkl}}$ são as variáveis independentes; $\mu$ é a média geral das observações; $\mathrm{T}_{\mathrm{i}}$ é o efeito do tratamento de ordem i, em que 1 é o desmame precoce, 2 é a amamentação controlada e 3 é o desmame convencional; $\mathrm{P}_{\mathrm{j}}$ é o efeito de período de ordem $\mathrm{j}$, em que 1 são os bezerros nascidos em junho e 2 são os bezerros nascidos em julho; $\mathrm{C}_{\mathrm{k}}$ é o efeito da categoria da vaca de ordem $\mathrm{k}$, em que 1 é jovem, 2 é adulta e 3 é velha; $S_{1}$ é o efeito do sexo do bezerro de ordem 1 , em que 1 é macho e 2 é fêmea; $(\mathrm{T} * \mathrm{C})_{\mathrm{ik}}$ é o efeito da interação entre o i-ésimo tratamento com a k-ésima categoria da vaca; e $\varepsilon_{\mathrm{ijkl}}$ é o erro aleatório residual.

\section{Resultados e Discussão}

As vacas apresentaram bom ECC durante a estação de nascimento, de aproximadamente 3,5 pontos, o que pode ser explicado pela boa qualidade da pastagem nesse período; no entanto, não houve diferença significativa entre os diferentes manejos de amamentação (Tabela 2).

Observou-se perda de peso e de ECC dos animais, do parto até a estação de monta (Tabela 2). Após o início da estação de monta, houve aumento de peso das vacas em todos os tratamentos, mesmo sob condições de disponibilidade e qualidade inferior de forragem. Uma possível explicação para isso é que, durante esse período, o pico de produção de leite se estabelece semanas antes do pico de ingestão de matéria seca, e a energia utilizada para a manutenção e a produção de leite é maior do que a obtida com a alimentação (Sartori \& Guardieiro, 2010).

Nos primeiros 30 dias após o desmame, as vacas do tratamento desmame precoce apresentaram menor ECC $(3,42$ pontos $)$ do que as do tratamento amamentação controlada (3,65 pontos), o que pode ter sido resultado do estresse causado pelo desmame precoce no início da estação de monta (Tabela 2). Porém, aos 60 dias de estação de monta, observou-se melhora no ECC, nas vacas do tratamento amamentação precoce, em razão do ganho de peso a partir dos 30 dias. Já as vacas submetidas ao tratamento amamentação controlada apresentaram ganho de peso e ECC similares aos das vacas do tratamento desmame convencional. Isso indica mudança na partição dos nutrientes, quando interrompida a amamentação, em consequência da diminuição das exigências nutricionais, o que permitiu que as vacas recuperassem o ECC. Melhoria no ECC em vacas submetidas ao desmame precoce também foi relatada por Fagundes et al. (2003).

As vacas com amamentação controlada conceberam 11 dias antes do que as do desmame convencional a partir do início da estação de monta (37,69 contra 49,55 dias, respectivamente). Esse resultado pode ser explicado pela possível elevação da concentração de opioides endógenos (encefalinas, endorfinas e dinorfinas) na circulação sanguínea das vacas com desmame precoce. Em vacas que amamentam, esses hormônios atuam diretamente na inibição dos neurônios responsáveis pela liberação de gonadotrofina $(\mathrm{GnRH})$ e também suprimem a liberação de hormônio luteinizante (LH), ao agirem na pituitária anterior (Williams \& Griffth, 1995). Assim, com a remoção do bezerro, o eixo hipotálamo-hipofisário da vaca é ativado, o que provoca aumento da frequência dos pulsos do $\mathrm{LH}$ e da concentração de IGF-I e, consequentemente, diminuição do período de serviço (Kawashima et al., 2008). Vargas Junior et al. (2013) também constataram que a maior frequência de mamadas em bezerros Nelore é responsável pelo maior período de anestro nas vacas.

Deve-se ressaltar que a obtenção de maiores índices de concepção no início da estação de monta é importante, pois há concentração de maior número de partos no primeiro mês da estação de parição e mais tempo para recuperação da reserva corporal das vacas até o início da estação de monta subsequente (Grecellé et al., 2006). Independentemente do tratamento, as vacas avaliadas no presente trabalho apresentaram ótimo índice de dias para concepção, com menos de 50 dias a partir do início da estação de monta. Novilhas e vacas que concebem e parem mais cedo, na respectiva época, produzem e desmamam maior número e massa

Pesq. agropec. bras., Brasília, v.49, n.1, p.49-56, jan. 2014 DOI: 10.1590/S0100-204X2014000100007 
$(\mathrm{kg})$ de terneiros durante sua vida produtiva (Azeredo et al., 2007). Da mesma forma, vacas que parem mais tarde tendem a parir mais tarde no próximo ano, ou a não parir.

A taxa de prenhez não foi influenciada pelos tratamentos (Tabela 2); porém, a taxa reprodutiva foi bastante alta em todos eles. A menor média observada, nas vacas do desmame convencional, chegou a $90 \%$, enquanto na amamentação controlada e no desmame precoce, os valores foram de 95 e $97 \%$, respectivamente. Esses dados comprovam a importância da manutenção de vacas em boas condições nutricionais durante o período de parto e pós-parto. Os animais avaliados chegaram ao início da estação de monta com ECC acima de 3,10 pontos. Mesmo com a estação de monta em época de entressafra das pastagens - fim de inverno e início de primavera - alcançou-se índice de fertilidade acima de $90 \%$.

Segundo o National Research Council (2001), uma vaca de grande porte, com pico de lactação de $8 \mathrm{~kg}$ por dia, exige 4,13 Mcal de energia líquida por dia, para produção de leite no terceiro, no quarto e no quinto mês de lactação. A mantença mais a gestação necessitam de 10,28 Mcal de energia líquida por dia, ou seja, nesse período, a exigência energética de uma vaca submetida ao desmame precoce é aproximadamente $30 \%$ menor.
Mesmo quando se manteve o bezerro em amamentação contínua, as vacas apresentaram alta taxa de prenhez (90\%) (Tabela 2) uma vez que, em razão da sua boa condição corporal, não houve necessidade de poupar energia com o desmame precoce dos bezerros. De acordo com Vieira et al. (2005), é possível obter alta taxa de prenhez em vacas em regime de pastejo, desde que elas estejam com escore de condição corporal acima de 3 pontos. Porém, nos casos em que as vacas sofrem restrição alimentar, a amamentação provoca efeito sobre a reativação do ciclo reprodutivo desses animais, como observado por Pencai et al. (2011), que relataram diferença de 32,8 e $30,6 \%$ de taxa de prenhez em vacas submetidas à amamentação controlada $(87,35)$ e ao desmame precoce $(85,1 \%)$, em comparação às vacas submetidas ao desmame convencional $(54,5 \%)$.

Em todos os tratamentos, constatou-se média de ganho diário de peso durante a estação de monta de $0,5 \mathrm{~kg}$ por vaca por dia e ganho de ECC de 0,54 pontos (Tabela 2). Portanto, evidencia-se que a disponibilidade nutricional foi suficiente para favorecer o ganho de peso e de escore corporal e o acúmulo de adipócitos, que desencadeiam o efeito de hormônios metabólicos $(\mathrm{GH}$, insulina, IGF-I e II, e leptina) no aumento da frequência do pulso do LH (Souza et al., 2009), o que, por sua vez, resultou em taxas de prenhez acima de $90 \%$.

Tabela 2. Médias \pm erro-padrão, coeficiente de variação e significância estatística ( $p>F)$ para as características de desempenho de vacas de corte Purunã, submetidas a diferentes manejos de amamentação(1).

\begin{tabular}{|c|c|c|c|c|c|}
\hline Período & Desmame precoce & Amamentação controlada & Desmame convencional & CV $(\%)$ & $\mathrm{p}>\mathrm{F}$ \\
\hline & \multicolumn{5}{|c|}{ Peso $(\mathrm{kg})$} \\
\hline Ao parto & $430,00 \pm 6,41$ & $437,00 \pm 6,72$ & $426,00 \pm 6,13$ & 10,54 & 0,7140 \\
\hline Início da estação de monta & $414,76 \pm 6,79$ & $426,25 \pm 7,01$ & $405,57 \pm 6,39$ & 11,72 & 0,3475 \\
\hline 30 dias de estação de monta & $439,94 \pm 7,75$ & $458,90 \pm 7,92$ & $445,14 \pm 7,25$ & 11,99 & 0,1912 \\
\hline 60 dias de estação de monta & $462,91 \pm 7,07$ & $473,55 \pm 7,18$ & $458,73 \pm 6,57$ & 10,40 & 0,6413 \\
\hline \multirow[t]{2}{*}{ Final da estação de monta } & $469,02 \pm 6,85$ & $467,38 \pm 6,96$ & $448,8 \pm 6,37$ & 10,14 & 0,4572 \\
\hline & \multicolumn{5}{|c|}{ Escore de condição corporal (pontos) } \\
\hline Ao parto & $3,38 \pm 0,04$ & $3,46 \pm 0,04$ & $3,37 \pm 0,04$ & 9,28 & 0,3645 \\
\hline Início da estação de monta & $3,11 \pm 0,05$ & $3,25 \pm 0,05$ & $3,12 \pm 0,05$ & 10,89 & 0,0799 \\
\hline 30 dias de estação de monta & $3,42 \pm 0,05 b$ & $3,65 \pm 0,05 b$ & $3,56 \pm 0,05 \mathrm{ab}$ & 8,99 & 0,0088 \\
\hline 60 dias de estação de monta & $3,62 \pm 0,05$ & $3,75 \pm 0,05$ & $3,67 \pm 0,04$ & 8,91 & 0,2471 \\
\hline \multirow[t]{2}{*}{ Final da estação de monta } & $3,68 \pm 0,05$ & $3,79 \pm 0,05$ & $3,59 \pm 0,04$ & 8,61 & 0,0372 \\
\hline & \multicolumn{5}{|c|}{ Ganho de peso $(\mathrm{kg})$} \\
\hline Parto ao início da estação de monta & $-17,20 \pm 4,58$ & $-11,39 \pm 4,73$ & $-20 \pm 4,35$ & $-106,85$ & 0,2253 \\
\hline GMD durante a estação de monta & $0,66 \pm 0,06$ & $0,49 \pm 0,06$ & $0,50 \pm 0,06$ & 76,36 & 0,0522 \\
\hline \multirow[t]{2}{*}{ Ganho de peso total durante a estação de monta } & $56,23 \pm 5,09$ & $42,09 \pm 5,17$ & $42,23 \pm 4,73$ & 76,32 & 0,0543 \\
\hline & \multicolumn{5}{|c|}{ Desempenho reprodutivo } \\
\hline Concepção na estação de monta (dias) & $41,06 \pm 2,60 \mathrm{ab}$ & $37,69 \pm 2,63 b$ & $49,55 \pm 2,48 \mathrm{a}$ & 85,00 & 0,0204 \\
\hline Taxa de prenhez $(\%)$ & $97 \pm 0,03$ & $96 \pm 0,03$ & $90 \pm 0,03$ & 32,00 & 0,3336 \\
\hline
\end{tabular}

${ }^{(1)}$ Médias seguidas por letras iguais não diferem, entre si, pelo teste de Tukey, a 5\% de probabilidade. GMD, ganho médio diário. 
Esse resultado é indicativo de que o efeito da amamentação decorre, principalmente, da menor exigência energética da vaca, provocada pela menor sucção de leite, o que favorece a retomada da condição corporal, fator determinante para o retorno da atividade cíclica da vaca no pós-parto (Flores et al., 2007). Pötter \& Lobato (2004) também verificaram taxas de prenhez semelhantes, de 97,8 e 91,3\%, para os tratamentos desmame precoce e desmame convencional, respectivamente, motivadas pelas boas condições nutricionais e fisiológicas no pós-parto.

Ao se avaliar a produção de bezerros dentro de cada tratamento (Tabela 3), observou-se que as vacas do tratamento desmame precoce apresentaram a menor produção de massa $(\mathrm{kg})$ de bezerros por vaca $(132,83 \mathrm{~kg})$, seguidas das vacas do tratamento desmame convencional $(145,40 \mathrm{~kg})$ e das vacas do tratamento amamentação controlada, as quais apresentaram a maior média $(159,06 \mathrm{~kg})$. Portanto, as vacas dos tratamentos amamentação controlada e desmame convencional tiveram maior eficiência reprodutiva (35,09 e $35,34 \mathrm{~kg}$, respectivamente) que as do desmame precoce $(28,26 \mathrm{~kg})$.

A menor eficiência reprodutiva das vacas do desmame precoce decorre do peso do bezerro $(132,83 \mathrm{~kg}$ por vaca) ao final do período reprodutivo, que foi menor nesse tratamento. Portanto, quando as vacas são mantidas em boas condições nutricionais e corporais ao parto e apresentam altas taxas de prenhez $(>90 \%)$, a utilização do desmame precoce torna-se inadequado, em termos de eficiência reprodutiva.

A eficiência produtiva ao parto das vacas do desmame precoce $(31 \mathrm{~kg}$ de bezerros por $100 \mathrm{~kg}$ de vaca), aos 150 dias de idade dos bezerros, embora tenha sido a menor entre os tratamentos, foi similar à obtida por McManus et al. (2002), para bezerros Nelore desmamados aos 210 dias $(34,78 \mathrm{~kg})$, mantidos em pastagem natural e cultivada. Dessa forma, pode-se observar que o desmame precoce apresenta eficiência produtiva similar à de vacas em desmame convencional, de diferentes raças e ambientes.

A eficiência reprodutiva, expressa em $\mathrm{kg}$ de bezerro por $100 \mathrm{~kg}$ de vaca, foi semelhante entre os sistemas de manejo com amamentação controlada e desmame convencional, ambos com valores superiores ao do sistema de desmame precoce (Tabela 3). O mesmo foi verificado quanto à eficiência reprodutiva ao parto e ao final do período reprodutivo. Entretanto, o índice de produção de bezerros, expresso em $\mathrm{kg}$ de bezerro por vaca, foi maior para o sistema de manejo com amamentação controlada, e não houve diferença entre o desmame precoce e o convencional. $\mathrm{O}$ aumento do índice de produção de bezerros ( $\mathrm{kg}$ de bezerro por vaca) pode ter sido influenciado pela maior taxa de prenhez (96\%) das vacas do tratamento com amamentação controlada, uma vez que esta é utilizada para o cálculo do referido índice. Esse resultado está de acordo com o obtido por Vaz et al. (2010a), que relataram que, quanto maiores as taxas de prenhez e desmame, maior a produção em $\mathrm{kg}$ de bezerro por $\mathrm{kg}$ de vaca.

As vacas submetidas ao desmame precoce apresentaram menor eficiência produtiva, tanto no parto como no desmame, do que às dos outros dois tratamentos (Tabela 3), em razão do menor peso dos bezerros ao desmame, na idade convencional, o que foi observado no índice de produção de bezerro $(132,83 \mathrm{~kg})$. O tratamento amamentação controlada não afetou os índices de eficiência produtiva, que foram similares aos das vacas do desmame convencional, tanto na eficiência ao parto como ao desmame.

Em outras condições ambientais, os resultados obtidos para o desmame precoce, em comparação ao convencional, podem ser inversos aos encontrados no presente trabalho. Em pastagens naturais e em

Tabela 3. Médias \pm desvio-padrão, coeficiente de variação e probabilidade $(p>F)$ para eficiência produtiva e reprodutiva de vacas de corte Purunã submetidas a tratamentos de manejo de amamentação ${ }^{(1)}$.

\begin{tabular}{|c|c|c|c|c|c|}
\hline Característica & $\begin{array}{c}\text { Desmame } \\
\text { precoce }\end{array}$ & $\begin{array}{c}\text { Amamentação } \\
\text { controlada }\end{array}$ & $\begin{array}{c}\text { Desmame } \\
\text { convencional }\end{array}$ & $\mathrm{CV}$ & $\mathrm{p}>\mathrm{F}$ \\
\hline Îndice de produção de bezerros (kg de bezerros por vaca) ${ }^{(2)}$ & $132,83 \pm 2,77 \mathrm{c}$ & $159,06 \pm 2,89 \mathrm{a}$ & $145,40 \pm 2,64 b$ & 13,91 & 0,0001 \\
\hline Eficiência reprodutiva ( $\mathrm{kg}$ de bezerro por $100 \mathrm{~kg}$ de vaca) ${ }^{(3)}$ & $28,26 \pm 0,79 b$ & $35,09 \pm 0,79 \mathrm{a}$ & $35,34 \pm 0,73 \mathrm{a}$ & 16,38 & $<0,0001$ \\
\hline Eficiência produtiva ao parto $(\mathrm{kg} \text { de bezerro por } 100 \mathrm{~kg} \text { de } \mathrm{PVP})^{(4)}$ & $31,00 \pm 0,94 \mathrm{~b}$ & $38,41 \pm 0,98 \mathrm{a}$ & $38,63 \pm 0,90 \mathrm{a}$ & 18,56 & 0,0001 \\
\hline Eficiência produtiva ao FPR (kg PBFPR por $100 \mathrm{~kg}$ de PVD) ${ }^{(5)}$ & $29,13 \pm 0,81 \mathrm{~b}$ & $36,17 \pm 0,82 \mathrm{a}$ & $36,43 \pm 0,75 \mathrm{a}$ & 16,38 & 0,0397 \\
\hline
\end{tabular}

${ }^{(1)}$ Médias seguidas de letras iguais não diferem entre si, pelo teste de Tukey, a $5 \%$ de probabilidade. ${ }^{(2)}(\mathrm{PBFPR} \times \mathrm{TP}) / 100$. ${ }^{(3)}(\mathrm{PBFPR} / 100) \times 100 \times \mathrm{TP} / \mathrm{PVD}$. ${ }^{(4)} \mathrm{PBFPR} / 100 \times \mathrm{PVP} .{ }^{(5)}(\mathrm{PBFPR} / \mathrm{PVD}) \times 100$. FPR, final do período reprodutivo; PBFPR, peso do bezerro ao final do período reprodutivo (kg); TP, taxa de prenhez (\%); PVD, peso da vaca ao desmame (kg); PVP, peso da vaca ao parto $(\mathrm{kg})$. 
Urochloa humidicola, Vaz et al. (2010b) constataram que as vacas submetidas ao desmame precoce ( 76 dias de idade) apresentaram maior índice de produção de bezerro e eficiência produtiva do que as vacas do desmame convencional (148 dias). Porém, no presente trabalho, as condições nutricionais do rebanho indicam que é possível buscar altos índices produtivos e reprodutivos no rebanho de cria sem a necessidade da utilização do desmame precoce ou da amamentação controlada.

\section{Conclusões}

1. Os manejos com desmame precoce, amamentação controlada e desmame convencional não influenciam significativamente a taxa de prenhez de vacas de corte Purunã, mantidas em boas condições corporais e nutricionais durante a estação de reprodução.

2. A amamentação controlada diminui o período entre o parto e a próxima concepção, e o desmame precoce provoca queda na eficiência produtiva das vacas.

\section{Referências}

AZEREDO, D.M. de; ROCHA, D.C.; JOBIM, M.I.M.; MATTOS, R.C.; GREGORY, R.M. Efeito da sincronização e da indução de estros em novilhas sobre a prenhez e o índice de repetição de crias na segunda estação reprodutiva. Ciência Rural, v.37, p.201-205, 2007. DOI: 10.1590/S0103-84782007000100032.

BORGES, J.B.S.; GREGORY, R.M. Indução da atividade cíclica ovariana pós-parto em vacas de corte submetidas à interrupção temporária do aleitamento associada ou não ao tratamento com norgestomet-estradiol. Ciência Rural, v.33, p.1105-1110, 2003. DOI: $10.1590 / \mathrm{S} 0103-84782003000600016$.

FAGUNDES, J.I.B.; LOBATO, J.F.P.; SCHENKEL, F.S. Efeito de duas cargas animais em campo nativo e de duas idades à desmama no desempenho de vacas de corte primíparas. Revista Brasileira de Zootecnia, v.32, p.1722-1731, 2003. DOI: 10.1590/ S1516-35982003000700023.

FLORES, R.; LOOPER, M.L.; RORIE, R.W.; LAMB, M.A.; REITER, S.T.; HALLFORD, D.M., KREIDER, D.L.; ROSENKRANS JUNIOR, C.F. Influence of body condition and bovine somatotropin on estrous behavior, reproductive performance, and concentrations of serum somatotropin and plasma fatty acids in postpartum Brahman-influenced cows. Journal of Animal Science, v.85, p.1318-1329, 2007. DOI: 10.2527/jas.2006-606.

GODOY, M.M. de; ALVES, J.B.; MONTEIRO, A.L.G.; VALÉRIO FILHO, W.V. Parâmetros reprodutivo e metabólico de vacas da raça Guzerá suplementadas no pré e pós-parto. Revista Brasileira de Zootecnia, v.33, p.103-111, 2004. DOI: 10.1590/ S1516-35982004000100014.
GRECELLÉ, R.A.; BARCELLOS, J.O.J.; BRACINI NETO, J.; COSTA, E.C.; PRATES, E.R. Taxa de prenhez de vacas Nelore X Hereford em ambiente subtropical sob restrição alimentar. Revista Brasileira de Zootecnia, v.35, p.1423-1430, 2006. DOI: 10.1590/ S1516-35982006000500023.

HAYDOCK, K.P.; SHAW, N.H. The comparative yield method for estimating dry matter yield of pasture. Australian Journal of Experimental Agriculture and Animal Husbandry, v.15, p.663-670, 1975. DOI: 10.1071/EA9750663.

KAWASHIMA C.; KIDA, K.; MATSUHASHI, M.; MATSUI, M.; SHIMIZU, T.; MATSUNAGA, N.; ISHII, M.; MIYAKE, Y.; MIYAMOTO, A. Effect of suckling on the reproductive performance and metabolic status of obese Japanese Black cattle during the early postpartum period. Journal of Reproduction and Development, v.54, p.46-51, 2008. DOI: 10.1262/jrd.19097.

LOWMAN, B.G.; SCOTT, N.; SOMERVILlE, S. Condition scoring beef cattle. Edinburgh: East of Scotland College of Agriculture, 1973. 8p. (Bulletin, 6).

MCMANUS, C.; SAUERESSIG, M.G.; FALCÃO, R.A.; SERRANO, G.; MARCELINO, K.R.A.; PALUDO, G.R. Componentes reprodutivos e produtivos no rebanho de corte da Embrapa Cerrados. Revista Brasileira de Zootecnia, v.31, p.648-657, 2002. DOI: 10.1590/S1516-35982002000300015.

MENEGAZ, A.L.; LOBATO, J.F.P.; PEREIRA, A.C.G. Influência do manejo alimentar no ganho de peso e no desempenho reprodutivo de novilhas de corte. Revista Brasileira de Zootecnia, v.37, p.1844-1852, 2008. DOI: 10.1590/S1516-35982008001000019.

NATIONAL RESEARCH COUNCIL. Nutrient requirements of dairy cattle. $7^{\text {th }}$ ed. Washington: National Academic Press, 2001. $381 \mathrm{p}$.

PENCAI, F.W.; KOZICK, L.E.; COSTA, C.E.M.P. da; SILVA, N.L. da; MOLETTA, J.L.; MOTTA, J.B. de O. Indução ao estro pós puerperal em bovinos mestiços de corte mediante o emprego de diferentes protocolos de amamentação. Veterinária e Zootecnia, v.18, p.53-62, 2011.

PÖTTER, B.A.A.; LOBATO, J.F.P. Efeitos de carga animal, pastagem melhorada e da idade de desmame no comportamento reprodutivo de vacas primíparas. Revista Brasileira de Zootecnia, v.33, p.192-202, 2004. DOI: 10.1590/S1516-35982004000100023.

SARTORI, R.; GUARDIEIRO, M.M. Fatores nutricionais associados à reprodução da fêmea bovina. Revista Brasileira de Zootecnia, v.39, p.422-432, 2010. DOI: 10.1590/ S1516-35982010001300047.

SOUZA, F.A.; CANISSO, I.F.; BORGES, A.M.; VALE FILHO, V.R.; LIMA, A.L.; SILVA, E.C. Restrição alimentar e os mecanismos endócrinos associados ao desenvolvimento folicular ovariano em vacas. Revista Brasileira de Reprodução Animal, v.33, p.61-65, 2009.

VARGAS JUNIOR, F.M. de.; WECHSLER, F.S.; OLIVEIRA, M.V.M. de.; SENO, L. de O.; FERNANDES, A.R.M.; CAMILO, F.R. Reproductive efficiency of Nellore cows nursing Nellore or crossbred Simmental $\times$ Nellore calves. Revista Brasileira de Zootecnia, v.42, p.475-480, 2013. DOI: 10.1590/ S1516-35982013000700003. 
VAZ, R.Z.; LOBATO, J.F.P.; RESTLE, J. Influence of weaning age on the reproductive efficiency of primiparous cows. Revista Brasileira de Zootecnia, v.39, p.299-307, 2010a. DOI: 10.1590/ S1516-35982010000200011.

VAZ, R.Z.; LOBATO, J.F.P.; RESTLE, J. Productivity and efficiency of cow herds submitted to two weaning ages. Revista Brasileira de Zootecnia, v.39, p.1849-1856, 2010b. DOI: 10.1590/ S1516-35982010000800030.

VIEIRA, A.; LOBATO, J.F.P.; TORRES JUNIOR, R.A. de A.; CEZAR, I.M.; CORREA, E.S. Fatores determinantes do desempenho reprodutivo de vacas Nelore na região dos Cerrados do Brasil Central. Revista Brasileira de Zootecnia, v.34, p.2409-2416, 2005. DOI: 10.1590/ S1516-35982005000700029.

WEISS, W.P.; CONRAD, H.R.; PIERRE, N.R.A theoretically-based model for predicting total digestible nutrient values of forages and concentrates. Animal feed Science and Technology, v.39, p.95-110, 1992. DOI: 10.1016/0377-8401(92)90034-4.

WILLIAMS, G.L.; GRIFFITH, M.K. Sensory and behavioural control of gonadotrophin secretion during suckling-mediated anovulation in cows. Journal of Reproduction and Fertility, v.49, p.463-475, 1995.

Recebido em 1ํ de outubro de 2013 e aprovado em 27 de dezembro de 2013 